\title{
Superior Mesenteric Artery Thrombosis in a Patient with COVID-19: A Unique Presentation
}

\author{
Burcin Agridag Ucpinar and Cennet Sahin \\ Department of Radiology, University of Health Sciences, Istanbul Sisli Hamidiye Etfal Training and Research Hospital, İstanbul, Turkey
}

\begin{abstract}
Coronavirus disease-2019 (COVID-19) characteristics and clinical spectrum are currently evolving with the collaboration of clinicians.Arterial thromboembolic events due to COVID-19 are extremely rare; and all reported cases so far were of ischemic strokes and pulmonary embolism. In this case report, we present a case of superior mesenteric artery (SMA) thrombosis in a patient of COVID-19 pneumonia. To our knowledge, this is the first case that demonstrates acute mesenteric ischemia in a patient with COVID-19 pneumonia.
\end{abstract}

Key Words: COVID-19, Mesenteric ischemia, Thrombosis.

How to cite this article: Ucpinar BA, Sahin C. Superior Mesenteric Artery Thrombosis in a Patient with COVID-19: A Unique Presentation. J Coll Physicians Surg Pak 2020; 30(JCPSPCR):CR112-CR114.

\section{INTRODUCTION}

The coronavirus disease 2019 (COVID-19) emerged from China in December 2019, but quickly spread throughout the world; and World Health Organisation (WHO) declared the infection as a global pandemic on $11^{\text {th }}$ March, 2020. The disease has infected more than two million people currently and caused more than 120,000 deaths, worldwide. ${ }^{1}$ The disease course tends to begin with fever, persistent dry cough, tiredness, myalgia and difficulty in breathing. However, many other symptoms havebeen identified including headache, chest pain, loss of taste and smell sensation, and diarrhea. ${ }^{2}$ An unexpected course of this disease includes thrombosis-related complications, which have been identified in patients with COVID-19, especially in intensive care unit (ICU) patients. After the detection of these thrombosis events, thromboprophylaxis is a main component of COVID-19 treatment, in addition to other antiviral and antimalarial medications. The thrombotic events are important cause of death in patients with COVID-19. ${ }^{3}$

There are reports in the literature about venous thromboembolism and recommendations on thromboprophylaxis. However, reported arterial thrombosis events in COVID-19 cases are rare; and those reported cases were all of ischemic strokes and pulmonary embolism. ${ }^{4,5}$

Correspondence to: Dr. Burcin Agridag Ucpinar, Department of Radiology, University of Health Sciences, Istanbul Sisli Hamidiye Etfal Training and Research Hospital, İstanbul, Turkey

E-mail:drburcinagridag@gmail.com

Received: April 21, 2020; Revised: April 25, 2020;

Accepted: April 25, 2020

DOI: https://doi.org/10.29271/jcpsp.2020.JCPSPCR.CR112
Systemic arterial thrombosis events, besides intracranial involvement, have not been reported in the literature.

Herein, we report a case of superior mesenteric artery (SMA) thrombosis in a patient admitted dueto COVID-19 pneumonia. To our knowledge, this is the first case of extracranial artery thrombosis in a patient with COVID-19, which involved SMA.

\section{CASE REPORT}

An 82-year female patient was admitted to the Emergency Department withfever and persistentcough. On physical examination, temperature of the patient was $38.2^{\circ} \mathrm{C}$, heart and respiration rates were 72 beats and 26 breaths per minute, respectively. Blood pressure was $100 / 70 \mathrm{mmHg}$. The patient had a history of atrial fibrillation (AF) for over 4 years, chronic kidney disease (CKD) and hypertension. Current medications of the patient for the treatment of AF with accompanying CKD included enoxaparin $(0.4 \mathrm{cc}$, twice daily, subcutaneously), amlodipine (5 mg, twice daily) and metoprolol (50 mg, four times daily). Complete blood count and biochemical analysis were obtained. Due to the current ongoing pandemic, COVID-19 was suspected and real-time reverse transcriptase-polymerase chain reaction (rRT-PCR) test was performed onnasopharyngeal swab. A chest computerised tomography (CT) scan was obtained, due to old age and presence of multiple comorbidities. The CT scan revealed widespread bilateral ground-glass opacities (GGOs), predominantly in lower lobes with peripheral distribution (Figure 1). The patient was admitted to the inpatient COVID-19 ward with a presumptive diagnosis of COVID-19 pneumonia. Appropriate treatment with hydroxychloroquine, oseltamivir and ceftriaxone was started. The rRT-PCR results confirmed COVID-19infection. Enoxaparin administration was continued, due to ongoing AF and increased risk of thromboembolic events in COVID-19 patients. 


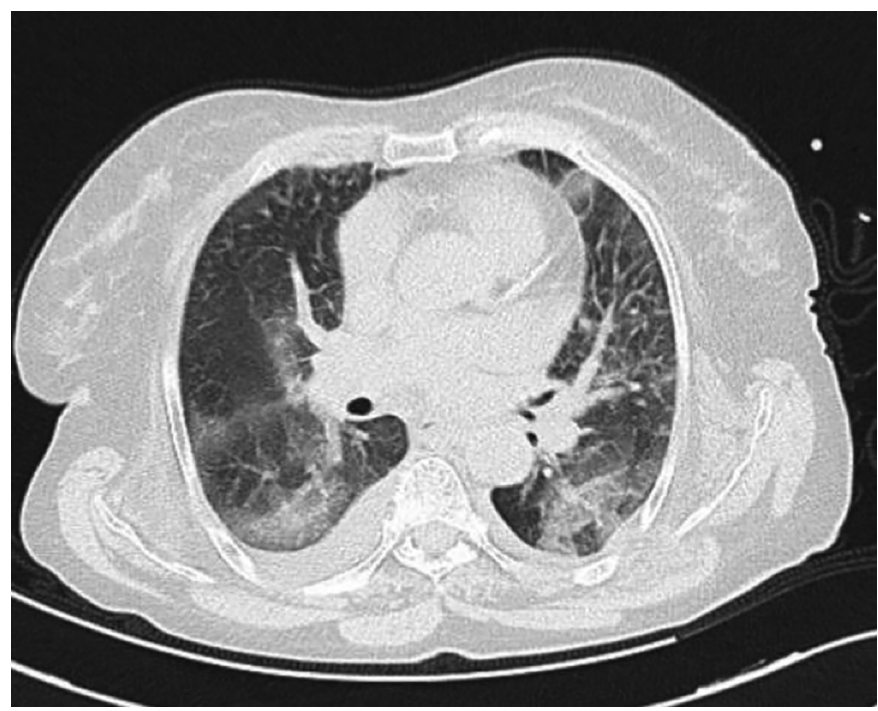

Figure 1: Axial CT of chest. Widespread ground-glass opacities are seen in peripheral distribution; accompanying pleural effusion is also present.

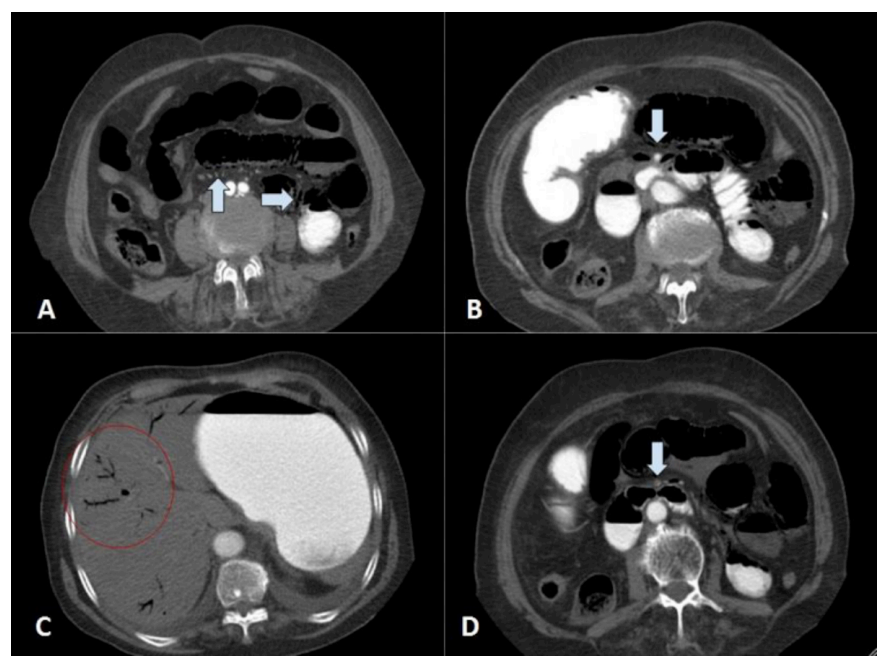

Figure 2: Contrast-enhanced CT scan of abdomen. A. Small bowel loops are dilated and submucosal air is seen in the non-dependent portion of the bowel walls. Air is also identified in mesenteric vessels (arrows). B. Proximal segment of the superior mesenteric artery is shown with arrow. There is contrast noted in the lumen (arrow). C. Linear structures with air inside are seen in the periphery of the liver which represents portal venous gas (red circle). D. Distal segment of the superior mesenteric artery is shown with luminal narrowing by intramural thrombosis (arrow).

On the $3^{\text {rd }}$ day of admission, sudden-onset abdominal pain and distention were reported by the patient. Laboratory investigations showed leukocytosis (WBC: $14.8 \times 10^{9} / \mathrm{L}$ ), Iymphopenia $\left(0.87 \times 10^{9} / \mathrm{L}\right)$ and increased lactate $(5.1 \mathrm{mmol} / \mathrm{L})$, D-dimer (1600 ng/mL) and C-reactive protein (196 mg/L). On physical examination, generalised peritoneal irritation signs were detected and a contrast enhanced CT scan of the abdomen was performed, to rule out possible causes of acute abdomen. On CT examination, small bowel segments were distended with diffuse submucosal pneumatosis (Figures 2A, 2B) and portomesenteric venous gas (Figure 2C). SMA thrombosis was identified (Figure 2D). Meanwhile, bedside echocardiography was performed to exclude atrial thrombus; and nothing remarkable was observed except for mild cardiomegaly.
Aggressive fluid resuscitation was initiated and the patient and her family were informed about the necessity of exploratory laparotomy. However, the patient developed cardiopulmonary arrest during preparation for surgery in the ward and did not respond to appropriate cardiopulmonary resuscitation attempts.

\section{DISCUSSION}

COVID-19 has become a worldwide problem, affecting every country on Earth. ${ }^{1}$ As healthcare workers and radiologists, our perspective on this disease is currently evolving with the collaboration of many radiologists across the world. Interpretation and reporting of thoracic imaging in COVID-19 patients are evolving and being continuously updated, as more cases are being followed, treated and reported. The COVID-19 primarily affects lungs; however, systemic thromboembolic events are increasingly recognised among COVID-19 cases. These events include pulmonary embolism, deep vein thrombosis of the leg, catheter-related thrombotic events and ischemic strokes. ${ }^{6}$ As radiologists, we should be aware of probability of these events in COVID-19 patients and evaluate the performed imagings, accordingly.

In patients with COVID-19, CT imaging of the thorax is the main diagnostic tool to evaluate the extent of the disease in lung parenchyma, especially in elderly patients and patients with certain comorbidities. Additional imaging methods are considered in patients with other symptoms including persistent headache, abdominal pain, upper or lower extremity pain and swelling in extremities, which may be related to thromboembolic events. These imagings vary according to the site of the symptoms. However, asymptomatic minor thromboembolic events may go unrecognised and the rate of these events might be higher than reported. Additionally, critically ill patients, who require ventilation support, are admitted to ICU and followed under strict quarantine measures. Thus, when thrombotic events are suspected, obtaining new imagings like CT is especially difficult in this patient group. Klok et al. have reported their experience on thrombotic events in COVID-19 cases, in a recently published article. ${ }^{4}$ They have reported the results of 184 patients with COVID-19, who were being followed in ICU. They detected 28 venous and 3 arterial thrombotic events in these patients. Venous thrombotic events were mainly pulmonary embolism and all arterial events were ischemic strokes. They also stated the possible underestimation of thromboembolic events in this patient group, due to diminished access to imaging methods, due to strict quarantine measures. ${ }^{4}$ Among COVID-19 patients, increased levels of d-dimers and changes in coagulation markers like prothrombin time are an important sign of increased likelihood of thromboembolic events. Zhou et al. reported their case series in Wuhan, China and detected increased coagulopathy in $19 \%$ of their patients. ${ }^{5}$ However, specific thromboembolic events related to this increased coagulopathy were not reported.

SMA is the major arterial supply for the small intestine and ascending colon. Thus, thrombosis of SMA is a fatal pathology, 
which bears a high risk of morbidity and mortality. Patients commonly present with severe abdominal pain and nausea, vomiting and diarrhea may also accompany this finding. ${ }^{7} \mathrm{CT}$ angiography is the gold standard for diagnosis and has a sensitivity of $96 \%$ and specificity of $94 \%$ in detecting acute and chronic forms of mesenteric ischemia. ${ }^{8}$ Bowel wall hypoenhancement, dilation, thickening, mesenteric edema, pneumatosis, free intraperitoneal air, and air in portal venous system are possible findings that can be observed in CT angiography scan. ${ }^{8}$ When diagnosed, patients need immediate intervention with aggressive fluid resuscitation, anticoagulation medications and laparotomy, if peritoneal irritation signs are present. The intervention likely involves bowel resection and anastomosis, which may require creation of ostomies. Even if diagnosed and intervened early, the pathology itself bears a high risk of mortality. ${ }^{9}$

The occlusion of SMA can occur either as a thrombotic occlusion of the vessel by a pre-existing underlying pathology or occlusion of the vessel by an embolus from a distant source. ${ }^{10}$ SMA thrombosis occurs at the narrowing zones of the vessels. The cause of arterial lumen narrowing is most commonly due to atherosclerosis. The emboli generally originate from a thrombus located in the heart. Thrombi originating from the heart are associated with atrial arrhythmias, valvular disease, ventricular dilatation and myocardial infarction. Major accompanying factors predisposing to thrombosis are dehydration, hypercoagulable state and decreased cardiac output. ${ }^{10}$ In this case, the patient was on anticoagulation regimen due to ongoing AF. However, normal bedside echocardiography, increased D-dimer levels, and acute onset of the gastrointestinal symptoms after hospitalization for COVID-19 infection strengthen the possibility of arterial thrombosis as a complication of COVID-19 infection rather than embolism from heart. Unfortunately, as no autopsy was performed after the sudden death of the patient, we were unable to clearly demonstrate the origin of SMA thrombosis. In any case, we believe that clinicians should be aware of thrombosis-related complications in COVID-19 patients.

In conclusion, increased likelihood of thrombotic events in patients with COVID-19 can cause fatal complications. Even though the likelihood of venous thrombosis is higher, arterial thrombosis is possible and should be kept in mind by the physicians who are dealing with patients with COVID-19.

\section{PATIENT'S CONSENT:}

Patient's consent was taken orally from the family of the patient.

\section{CONFLICT OF INTEREST:}

The authors declared no conflict of interest.

\section{AUTHORS' CONTRIBUTION:}

BAU: Data acquisition and analysis, interpretation, drafting, final approval.

CS: Interpretation, critical revision, final approval.

\section{REFERENCES}

1. World Health Organization. Coronavirus disease (COVID-19) outbreak. Available at: http://www.who.int [Accessed on April 10, 2020].

2. Guan WJ, Ni ZY, Hu Y. Clinical characteristics of coronavirus disease 2019 in China N Engl J Med 2020; 382(18): 1708-20. doi: 10.1056/NEJMoa2002032.

3. Wang T, Chen R, Liu C, Liang W, Guan W, Tang R, et al. Attention should be paid to venous thromboembolism prophylaxis in the management of COVID-19. Lancet Haematol 2020; 7(5):e362-e363. doi: 10.1016/S23523026(20)30109-5.

4. Klok FA, Kruip MJHA, van der Meer NJM, Arbous MS, Gommers DAMPJ, Kant KM, et al. Incidence of thrombotic complications in critically ill ICU patients with COVID-19. Thromb Res 2020; 191: 145-7. doi.org/10.1016/j.thromres. 2020.04.013.

5. Zhou F, Yu T, Du R, Fan G, Liu Y, Liu Z, et al. Clinical course and risk factors for mortality of adult inpatients with COVID-19 in Wuhan, China: a retrospective cohort study. Lancet 2020; 395(10229):1054-62. doi: 10.1016/S01406736(20)30566-3.

6. Casini A, Alberio L, Angelillo-Scherrer A. Thromboprophylaxis and laboratory monitoring for in-hospital patients with COVID-19 - A Swiss consensus statement by the working party hemostasis. Swiss Med Wkly 2020; 150:w20247. doi: 10.4414/smw.2020.20247. eCollection 2020 Apr 6.

7. Franca E, Kosove J. Mesenteric artery thrombosis. [Updated 2020 Jan 15]. In: StatPearls [Internet]. Treasure Island (FL): Stat Pearls Publishing; 2020 Jan.

8. Olson MC, Fletcher JG, Nagpal P, Froemming AT, Khandelwal A. Mesenteric ischemia: What the radiologist needs to know. Cardiovasc Diagn Ther 2019; 9(Suppl 1):S74-87. doi:10.21037/cdt.2018.09.06.

9. Gupta PK, Natarajan B, Gupta H, Fang X, Fitzgibbons RJ Jr. Morbidity and mortality after bowel resection for acute mesenteric ischemia. Surgery 2011; 150(4):779-87. doi: 10.1016/j.surg.2011.07.079.

10. Acosta S. Mesenteric ischemia. Curr Opin Crit Care 2015; 21(2): 171-8. doi:10.1097/mcc.0000000000000189. 